\title{
Erratum to: Generation and characterization of virus-free reprogrammed melanoma cells by the piggyBac transposon
}

\author{
Juan Yin · Yongna Fan · Dingxin Qin · \\ Xiaocui Bian $\cdot$ Xiaobao Bi
}

Published online: 17 July 2013

(c) Springer-Verlag Berlin Heidelberg 2013

\section{Erratum to: J Cancer Res Clin Oncol \\ DOI 10.1007/s00432-013-1431-3}

Unfortunately, in the original version of this paper, the authors have overlooked a mistake. The corresponding author for this paper is Juan Yin. Accordingly, the affiliation of corresponding author should be Department of Pathology, Institute of Basic Medical Sciences and School of Basic Medicine, Chinese Academy of Medical Sciences and Peking Union Medical College, Beijing 100005, China. Also, due to an oversight in manuscript preparation, the names of Yongna Fan, Dingxin Qin, and Xiaocui Bian were omitted. The missing information is supplied here.

The online version of the original article can be found under doi:10.1007/s00432-013-1431-3.

J. Yin $(\varangle) \cdot$ Y. Fan · D. Qin · X. Bian

Department of Pathology, Institute of Basic Medical Sciences and School of Basic Medicine, Chinese Academy of Medical Sciences and Peking Union Medical College,

Beijing 100005, China

e-mail: yinjuan1301323@gmail.com

\section{Bi}

Division of Structural Biology \& Biochemistry, School of Biological Sciences, Nanyang Technological University, 60 Nanyang Drive, Singapore 637551, Singapore 